\title{
Coprinopsis atramentaria extract, its organic acids, and synthesized glucuronated and methylated derivatives as antibacterial and antifungal agents
}

Sandrina A. Heleno ${ }^{\mathrm{a}, \mathrm{b}}$, Isabel C.F.R. Ferreira ${ }^{\mathrm{a},{ }^{*}}$, Ana Ćirićc ${ }^{\mathrm{c}}$, Jasmina Glamočlija ${ }^{\mathrm{c}}$, Anabela Martins $^{\mathrm{a}}$, Maria João R.P. Queiroz ${ }^{\mathrm{b}}$, Marina Sokovićc

${ }^{a}$ Centro de Investigação de Montanha, Escola Superior Agrária, Bragança, Portugal

${ }^{\mathrm{b}}$ Centro de Química, Universidade do Minho, Braga, Portugal

'University of Belgrade, Institute for Biological Research "Siniša Stanković", Department of Plant Physiology, Belgrade, Serbia

* Author to whom correspondence should be addressed (Isabel C.F.R. Ferreira; e-mail: iferreira@ipb.pt; telephone +351-273-303219; fax +351-273-325405). 


\begin{abstract}
Despite the available data regarding antimicrobial activity of phenolic acids, studies dealing with the effects of their metabolites or derivatives are scarce. Therefore, the antimicrobial and demelanizing activities of Coprinopsis atramentaria extract, its organic acids, and methylated and glucuronated derivatives were evaluated. The antifungal activity was stronger than the antibacterial effects. In general, individual compounds (mostly organic acids) gave higher activity than the extract and even higher than the standards used in the assays. Methylated derivatives presented the highest demelanizing activity toward Aspergillus niger, A. fumigatus and Penicillium verrucosum var. cyclopium). The inclusion of methyl groups in the parental compound (CoAM1, CoAM2 and CoAM3) strongly increased antibacterial and antifungal activities of CoA, while the inclusion of acetyl groups (CoAGP) increased the antifungal activity but the antibacterial properties were maintained. For HA and CA, the inclusion of methyl groups (HAM1, HAM2, HAM3 and CAM) increased the demelanizing activity, but decreased the antimicrobial properties. The present work contributes to the knowledge of the mechanisms involved in the antimicrobial properties of organic acids namely, phenolic acids, usually present in mushrooms. Organic acids, methylated and glucuronated derivatives could be used as antimicrobial agents.
\end{abstract}

Keywords: Antimicrobial activity, demelanizing activity, organic acids, methylated derivatives, glucuronated derivatives, wild mushroom 


\section{Introduction}

Global antibacterial resistance is an increasing public health problem due to the bacterial resistance developed to almost all the antibiotics. ${ }^{1}$ Natural resources have been exploited in the last years and among them, mushrooms could be an alternative source of new antimicrobials. $^{2}$

Although fungi are well known for the production of important antibiotic compounds (penicillins, streptomycins, rifamycins and others), the occurrence of antibiotics in mushrooms is less well documented. ${ }^{1}$ Nevertheless, the scientific community, searching for new therapeutic alternatives, studied many different species of mushrooms and has found antimicrobial effects. ${ }^{2-5}$

Lentinus edodes is the most studied species regarding antimicrobial properties and seems to have a broad activity against both gram-positive and gram-negative bacteria, ${ }^{2}$ and fungi. ${ }^{6}$ Nonetheless, the antimicrobial activity of wild species (mostly methanolic extracts) has also been reported, such as Lactarius deliciosus, ${ }^{7}$ Lepista nuda, ${ }^{8}$ Morchella esculenta $^{4}$ and Ganoderma lucidum. ${ }^{5}$

Coprinopsis atramentaria (Bull.: Fr.) Redhead, Vilgalys \& Moncalvo, is a wild edible mushroom previously characterized by us for its nutritional composition, and its methanolic extract showed antioxidant ${ }^{9}$ and antitumor activities. ${ }^{10} p$-Hydroxybenzoic (4.71 mg/100 g dry weight), $p$-coumaric $(0.82 \mathrm{mg} / 100 \mathrm{~g})$ and cinnamic $(1.70 \mathrm{mg} / 100 \mathrm{~g})$ acids were identified in the mentioned extract. Nonetheless, the mentioned compounds are rapidly metabolized in the human organism. Glucuronidation and methylation appears as prevalent metabolic pathways for phenolic acids in humans. ${ }^{11}$ 
So, despite dietary phenolic compounds being widely considered to contribute to health benefits in humans, little is known about the bioactive forms in vivo and the mechanisms by which they may contribute toward disease prevention. In fact, despite the available data concerning antimicrobial effects of phenolic acids, ${ }^{12-14}$ studies dealing with the antimicrobial activity of their metabolites or derivatives are scarce.

In the present work, it was evaluated and compared the antimicrobial and demelanizing activity of: i) $C$. atramentaria extract; ii) compounds identified in the extract: $p$ hydroxybenzoic, $p$-coumaric and cinnamic acids; iii) acetylated glucuronide derivatives (protected glucuronides) and iv) methylated derivatives, both prepared by chemical synthesis.

\section{Experimental}

\section{Wild mushroom}

Samples of Coprinopsis atramentaria (Bull.: Fr.) Redhead, Vilgalys \& Moncalvo were collected in Bragança (Northeast Portugal). After taxonomic identification of the sporocarps ${ }^{15}$ specimens were deposited at the herbarium of Escola Superior Agrária of Instituto Politécnico de Bragança. The samples were lyophilized (FreeZone 4.5 model 7750031, Labconco, Kansas, USA) and reduced to a fine dried powder (20 mesh).

\section{Preparation of the extract}

The powder $(\sim 10 \mathrm{~g})$ was extracted with methanol $(250 \mathrm{~mL})$ at $-20{ }^{\circ} \mathrm{C}$ for $6 \mathrm{~h}$. The extract was sonificated for $15 \mathrm{~min}$, centrifuged at $4000 \mathrm{~g}$ for $10 \mathrm{~min}$ and filtered through Whatman No.4 paper. The residue was then re-extracted with three additional $150 \mathrm{~mL}$ 
portions of methanol. The combined extracts were evaporated (rotary evaporator Büchi R-210; Flawil, Switzerland) at $40{ }^{\circ} \mathrm{C}$ to dryness.

\section{Compounds tested}

$p$-Hydroxybenzoic, $p$-coumaric and cinnamic acids (related to phenolic acids) were identified in $C$. atramentaria extract. ${ }^{9}$ For the antimicrobial assays, these compounds (Figure 1) were purchased from Sigma (St. Louis, MO, USA).

Methylated and glucuronated derivatives (Figure 1) were synthesized and completely characterized as described previously by the authors, ${ }^{10}$ and used in the antimicrobial assays.

\section{Antibacterial activity}

The following Gram-negative bacteria: Escherichia coli (ATCC 35210), Pseudomonas aeruginosa (ATCC 27853), Salmonella typhimurium (ATCC 13311), Enterobacter cloacae (ATCC 35030), and Gram-positive bacteria: Staphylococcus aureus (ATCC 6538), Bacillus cereus (clinical isolate), Micrococcus flavus (ATCC 10240), and Listeria monocytogenes (NCTC 7973) were used. The microorganisms were obtained from the Mycological laboratory, Department of Plant Physiology, Institute for biological research "Siniša Stanković", University of Belgrade, Serbia.

The minimum inhibitory (MIC) and minimum bactericidal (MBC) concentrations were determined by the microdilution method. Briefly, fresh overnight culture of bacteria was adjusted by the spectrophotometer to a concentration of $1 \times 10^{5} \mathrm{CFU} / \mathrm{mL}$. The requested $\mathrm{CFU} / \mathrm{mL}$ corresponded to a bacterial suspension determined in a spectrophotometer at 
$625 \mathrm{~nm}$ (OD 625). Dilutions of inocula were cultured on solid medium to verify the absence of contamination and check the validity of the inoculum. Extract and compounds tested were carried out in different dilution over the wells containing $100 \mu \mathrm{L}$ of Tryptic Soy Broth (TSB) and afterwards, $10 \mu \mathrm{L}$ of inoculum was added to all the wells. The microplates were incubated for $24 \mathrm{~h}$ at $37^{\circ} \mathrm{C}$. The MIC of the samples was detected following the addition of $40 \mu \mathrm{L}$ of iodonitrotetrazolium chloride (INT) $(0.2 \mathrm{mg} / \mathrm{mL})$ and incubation at $37^{\circ} \mathrm{C}$ for $30 \mathrm{~min}$. The lowest concentration that produced a significant inhibition (around 50\%) of the growth of the bacteria in comparison with the positive control was identified as the MIC. The minimum inhibitory concentrations (MICs) obtained from the susceptibility testing of various bacteria to tested extract were determined also by a colorimetric microbial viability assay based on reduction of a INT color and compared with positive control for each bacterial strains. ${ }^{16,17} \mathrm{MBC}$ was determined by serial sub-cultivation of $10 \mu \mathrm{L}$ into microplates containing $100 \mu \mathrm{L}$ of TSB. The lowest concentration that shows no growth after this sub-culturing was read as the MBC. Standard drugs, namely streptomycin and ampicillin were used as positive controls. 5\% DMSO was used as negative control.

\section{Antifungal activity}

For the antifungal bioassays, the following microfungi were used: Aspergillus fumigatus (ATCC 1022), Aspergillus ochraceus (ATCC 12066), Aspergillus versicolor (ATCC 11730), Aspergillus niger (ATCC 6275), Trichoderma viride (IAM 5061), Penicillium funiculosum (ATCC 36839), Penicillium ochrochloron (ATCC 9112) and Penicillium verrucosum var. cyclopium (food isolate). The organisms were obtained from the 
Mycological Laboratory, Department of Plant Physiology, Institute for Biological Research "Siniša Stanković", Belgrade, Serbia. The micromycetes were maintained on malt agar (MA) and the cultures were stored at $4{ }^{\circ} \mathrm{C}$ and sub-cultured once a month. ${ }^{18}$

The fungal spores were washed from the surface of agar plates with sterile $0.85 \%$ saline containing $0.1 \%$ Tween $80(\mathrm{v} / \mathrm{v})$. The spore suspension was adjusted with sterile saline to a concentration of approximately $1.0 \times 10^{5}$ in a final volume of $100 \mu \mathrm{L} /$ well. The inocula were stored at $4^{\circ} \mathrm{C}$ for further use. Dilutions of the inocula were cultured on solid MA to verify the absence of contamination and to check the validity of the inoculum.

Minimum inhibitory concentrations (MICs) determination was performed by a serial dilution technique using 96-well microtitre plates. The investigated extract and compounds were dissolved in 5\% solution of DMSO and added to broth malt medium with fungal inoculum. The microplates were incubated for $72 \mathrm{~h}$ at $28^{\circ} \mathrm{C}$. The lowest concentrations without visible growth (at the binocular microscope) were defined as MIC. The minimum fungicidal concentrations (MFCs) were determined by serial subcultivation of $2 \mu \mathrm{L}$ in microtitre plates containing $100 \mu \mathrm{L}$ of malt broth per well and further incubation for $72 \mathrm{~h}$ at $28^{\circ} \mathrm{C}$. The lowest concentration with no visible growth was defined as the MFC, indicating 99.5\% killing of the original inoculum. ${ }^{19}$ DMSO $5 \%$ was used as a negative control, while bifonazole and ketoconazole were used as positive controls.

\section{Demelanizing activity using micromycetes}

All the microfungi tested for antifungal activity of $C$. atramentaria methanolic extract and compounds were used to evaluate extract/compounds demelanizing activity. The 
micromycetes were maintained on malt agar and the cultures were stored at $4{ }^{\circ} \mathrm{C}$; 96 -well microliter plates were used. The fungal spores were washed from the surface of agar plates with sterile $0.85 \%$ saline containing $0.1 \%$ Tween $80(\mathrm{v} / \mathrm{v})$. The spore suspension was adjusted with sterile saline to a proximate concentration of $1.0 \times 10^{5}$ in a final volume of $100 \mu \mathrm{L} /$ well. Dilutions of the inocula were cultured on malt agar to verify the absence of contamination and to check the validity of the inoculum. Determination of minimum demelanizing concentrations (MDC) was performed by a serial dilution technique. The extract/compounds were dissolved in 5\% DMSO solution containing $0.1 \%$ Tween 80 $(v / v)(10 \mathrm{mg} / \mathrm{mL})$ and added in broth Malt medium with inoculum. The microplates were incubated at Rotary shaker $(160 \mathrm{rpm})$ for $72 \mathrm{~h}$ at $28^{\circ} \mathrm{C}$. A sample of mycelium was taken from the periphery of a colony grown on Malt extract medium enriched with different concentrations of tested extract. The samples were dried and fixed with lactophenol and observed under a light microscope (Microscope DMLS Typ 020518 500. Leica, Wetzlar. Neubauer Zählkammer. Eppendorf, Hamburg, Germany) to examine structural abnormalities. ${ }^{5}$ The lowest concentration that provoked demelanization of fungal hyphae and conidia was determined as MDC. Samples from the control plate without added extracts were also stained and observed. Solution of 5\% DMSO was used as a negative control.

\section{Results}

\section{Antibacterial activity}

The methanolic extract of $C$. atramentaria was active against all the tested bacteria with minimal inhibitory concentrations (MICs) of $1.0-2.0 \mathrm{mg} / \mathrm{mL}$ and bactericidal 
concentrations (MBCs) of $2.0-4.0 \mathrm{mg} / \mathrm{mL}$ (Table 1). The most resistant bacteria to the extract were Micrococcus flavus and Pseudomonas aeruginosa.

$p$-Coumaric acid $(\mathrm{CoA})$ showed activity against all the tested bacteria presenting MICs of $0.047-0.140 \mathrm{mg} / \mathrm{mL}$ and MBCs of $0.094-0.180 \mathrm{mg} / \mathrm{mL}$ being Bacillus cereus the most susceptible bacteria to this phenolic acid. CoA showed higher activity than streptomycin against all the bacteria except for Staphylococcus aureus and, higher activity than ampicillin for all the bacteria. CoAGP showed activity against all the studied bacteria presenting MICs of $0.047-0.140 \mathrm{mg} / \mathrm{mL}$ and MBCs of $0.094-0.375 \mathrm{mg} / \mathrm{mL}$. The most susceptible bacteria to this compound was Listeria monocytogenes and the most resistant were S. aureus and Escherichia coli. CoAGP revealed higher activity than streptomycin against almost all the bacteria except for $S$. aureus and E. coli and, higher activity than ampicillin for all the bacteria studied.

CoAM1 showed antibacterial activity with MICs of $0.020-0.125 \mathrm{mg} / \mathrm{mL}$ and MBCs of $0.065-0.250 \mathrm{mg} / \mathrm{mL}$, being B. cereus and L. monocytogenes the most susceptible bacteria, while $E$. coli was the most resistant. CoAM1 showed higher activity than streptomycin against all the bacteria except for S. aureus and, higher activity than ampicillin for all the bacteria. CoAM2 also revealed antibacterial activity against all the bacteria with MICs of $0.0312-0.125 \mathrm{mg} / \mathrm{mL}$ and MBCs of $0.0625-0.250 \mathrm{mg} / \mathrm{mL}$. The most susceptible bacteria to this compound were Salmonella typhimurium and Enterobacter cloacae, while the most resistant were E. coli and M. flavus. CoAM2 showed higher activity than the two standards for all the tested bacteria. CoAM3 also presented activity for all the studied bacteria with MICs of $0.0312-0.250 \mathrm{mg} / \mathrm{mL}$ and MBCs of $0.0625-0.500 \mathrm{mg} / \mathrm{mL}$. The most susceptible bacteria were $S$. aureus, B. cereus and E. cloacae, being P. aeruginosa 
and $E$. coli the most resistant ones. CoAM3 had higher activity than streptomycin against all bacteria, except $P$. aeruginosa and $E$. coli, and higher activity than ampicillin against all the bacteria.

Methylated derivatives of $p$-coumaric acid (CoAM1, CoAM2 and CoAM3) revealed higher activity than the parental compound ( $p$-coumaric acid), while for the glucuronide derivative (CoAGP), the antibacterial activity was maintained for almost all the bacteria.

HAM1 and HAM2 presented almost the same activity with MICs of 0.0625-0.200 $\mathrm{mg} / \mathrm{mL}$ and MBCs of $0.125-0.250 \mathrm{mg} / \mathrm{mL}$, being L. monocytogenes the most susceptible bacteria. HAM1 and HAM2 showed higher activity than streptomycin against almost all the tested bacteria except for S. aureus and B. cereus, and higher activity than ampicillin against all the bacteria. HAM3 also had antibacterial activity with MICs of 0.047-0.070 $\mathrm{mg} / \mathrm{mL}$ and MBCs of $0.094 \mathrm{mg} / \mathrm{mL}$. L. monocytogenes, P. aeruginosa, S. typhimurium and E. cloacae were the most susceptible bacteria to this compound. HAM3 revealed higher activity than the two standards against all the tested bacteria.

Methylated derivatives (HAM1, HAM2 and HAM3) presented lower antibacterial activity than the parental compound HA, but in most cases higher than the two studied standards.

CAM was active against all the tested bacteria with MICs of $0.0625-0.250 \mathrm{mg} / \mathrm{mL}$ and MBCs of $0.125-0.500 \mathrm{mg} / \mathrm{mL}$. L. monocytogenes, $P$. aeruginosa and E. cloacae were the most susceptible bacteria while $M$. flavus was the most resistant one. CAM revealed higher activity than streptomycin against almost all the bacteria except for $S$. aureus, $B$. cereus and $M$. flavus, and also higher activity than ampicillin for all the bacteria except 
for M. flavus. CA presented an excellent antibacterial activity against all the bacteria, and higher than its derivative, CAM, and much higher than the two standards.

All the compounds presented higher activity than C. atramentaria extract.

\section{Antifungal activity}

C. atramentaria methanolic extract revealed antifungal activity and all the tested compounds showed very high antifungal potential when compared with antifungal standards bifonazole and ketoconazole (Table 2). The extract presented MICs of 0.5-2.0 $\mathrm{mg} / \mathrm{mL}$ and minimum fungicidal concentrations (MFCs) of $1.0-4.0 \mathrm{mg} / \mathrm{mL}$. The highest activity was verified for Penicillium ochrochloron, while Aspergillus fumigatus was the most resistant fungi.

CoA was active against all the tested fungi with MICs of $0.0625-0.250 \mathrm{mg} / \mathrm{mL}$ and MFCs of $0.125-0.450 \mathrm{mg} / \mathrm{mL}$, being A. versicolor the most susceptible fungi while A. niger and P. funiculosum were the most resistant ones. CoA showed higher activity than bifonazole against A. versicolor, and than ketoconazole against the majority of the fungi. CoAGP showed a moderate activity with MICs of $0.014-0.056 \mathrm{mg} / \mathrm{mL}$ and MFCs of $0.125-0.250$ $\mathrm{mg} / \mathrm{mL}$. A. ochraceus, $P$. ochrochloron and $P$. verrucosum were the most susceptible fungi, while $A$. versicolor and $A$. niger were the most resistant fungi. CoAGP showed higher activity than ketoconazole against all the fungi and higher than befonazole against A. versicolor, $P$. ochrochloron and $P$. verrucosum.

CoAM1 showed antifungal activity presenting MICs of $0.015-0.150 \mathrm{mg} / \mathrm{mL}$ and MFCs of $0.0625-0.625 \mathrm{mg} / \mathrm{mL}$, being A. versicolor and P. ochrochloron the most susceptible fungi, while A. fumigatus was the most resistant one. This compound showed higher 
activity than the two standards against the majority of the tested fungi. CoAM2 showed an excellent activity presenting MICs of $0.0078-0.047 \mathrm{mg} / \mathrm{mL}$ and MFCs of $0.015-0.0625$ $\mathrm{mg} / \mathrm{mL}$; all the tested fungi were very susceptible to this compound with the exception of A. ochraceus and A. niger that were the most resistant ones. CoAM2 showed much higher antifungal activity than the two standards tested. CoAM3 presented MICs of 0.0078$0.120 \mathrm{mg} / \mathrm{mL}$ and MFCs of $0.015-0.450 \mathrm{mg} / \mathrm{mL}$, showing the best activity against $A$. versicolor and the lowest against A. niger. CoAM3 presented higher activity than the two standards against the majority of the fungi. All the p-coumaric acid derivatives, methylated (CoAM1, CoAM2 and CoAM3) and glucuronated (CoAGP) showed higher activity than the parental compound and in the most of the cases higher antifungal activity than the standards, particularly CoAM2 that presented an excellent activity.

HAM1 was active against all the tested fungi with MICs of $0.015-0.0625 \mathrm{mg} / \mathrm{mL}$ and MFCs of $0.0312-0.125 \mathrm{mg} / \mathrm{mL}$, being Tricholoma viride and P. funiculosum the most susceptible fungi, while $A$. niger was the most resistant fungi. HAM1 showed a much higher activity than the two standards against all the fungi. HAM2 was active with MICs of $0.0125-0.0625 \mathrm{mg} / \mathrm{mL}$ and MFCs of $0.0625-0.125 \mathrm{mg} / \mathrm{mL}$. The best activity was against $T$. viride, $P$. funiculosum and $P$. ochrochloron and the lowest activity was against A. niger. HAM2 showed higher activity than the two standards against all the tested fungi. HAM3 presented MICs of $0.0312-0.250 \mathrm{mg} / \mathrm{mL}$ and MFCs of $0.125-0.450 \mathrm{mg} / \mathrm{mL}$, being $P$. funiculosum and $P$. ochrochloron the most susceptible fungi, while $T$. viride was the most resistant one. This compound showed higher antifungal activity than the two standards against the majority of the tested fungi. All HA methylated derivatives showed 
higher activity than the two standards against all the tested fungi, but lowest activity than the parental compound.

CAM revealed a good activity against all the fungi with MICs of $0.015-0.047 \mathrm{mg} / \mathrm{mL}$ and MFCs of 0.0312-0.0625 mg/mL. A. versicolor, P. funiculosum and P. ochrochloron were the most susceptible fungi, while $A$. niger was the most resistant. CAM showed much higher antifungal activity than the two standards against all the fungi, but lower activity than the correspondent parental organic acid. All the compounds revealed antifungal activity in a range of MICs $0.0078-0.250 \mathrm{mg} / \mathrm{mL}$ and MFCs $0.0150-0.450 \mathrm{mg} / \mathrm{mL}$.

All the tested compounds presented higher antifungal activity than the C. atramentaria extract.

\section{Demelanizing activity}

Demelanizing activity of extract and individual compounds was evaluated toward the eight microfungi also used in antifungal activity assays; nevertheless the effects were significant for Aspergillus niger, A. fumigatus and Penicillium verrucosum var. cyclopium.

The extract and organic acid derivatives showed demelanizing effect on $A$. niger at concentration 0.030-0.100 mg/mL (example of HAM1 in Figure 2B; Figure 3A and 3B). Compounds HAM1 and HAM3 (Figure 2A; Figure 3C and D) showed demelanizing effect on A. fumigatus, lowering the amount of conidia and giving nude vesicle without conidia at concentration $0.030-0.100 \mathrm{mg} / \mathrm{mL}$. Demelanizing activity of CoAM1, CoAM2, HAM3 and CAM (Figure 2C and $\mathbf{3 E}$ and $\mathbf{3 F}$ ) was noticed on $P$. verrucosum at concentration of $0.005-0.05 \mathrm{mg} / \mathrm{mL}$, provoking fialides without conidia and lower 
numbers of conidia. Minimum demelanizing concentrations (MDC) were the lowest in the case of $P$. verrucosum among all other tested.

Minimum demelanizing concentrations are very close to the minimum inhibitory concentrations but slightly higher, which is marked on Figure 2 with arrows.

Morphological changes and demelanization of microfungi is presented on Figure 3. Changes in both Aspergillus species are obvious (Figure $\mathbf{3 A}$ and $\mathbf{3 C}$ ) and include depigmentation, morphological changes of conidiophores and small number of heads and nude vesicles, in comparison with untreated culture (Figure $\mathbf{3 B}$ and 3D). The demelanization and reduction of conidia numbers of Penicillium verrucosum and fialides without conidia is in contrast with the control mycelium, which has typical brush-like clusters and numerous free conidia. All of these were recorded under light microscope (Figure 3E and 3F).

\section{Discussion}

C. atramentaria methanolic extract showed antibacterial and antifungal activities against all the tested microorganisms. Osuji et al. ${ }^{20}$ also reported a good antibacterial activity of C. atramentaria methanolic extract (expressed in halos of inhibition zone), especially against gram-negative bacteria. Nevertheless, all the compounds exhibited very high antibacterial activity, much higher than $C$. atramentaria methanolic extract and, in most of the cases, higher than the two standards.

The antimicrobial activity of CoA was previously reported by Alves et al. ${ }^{14}$ against some of the herein tested bacteria such as E. coli and L. monocytogenes, but with lower effects, probably due to the different methodology used for the screening. 
For the glucuronide derivative of $p$-coumaric acid (CoAGP), the antibacterial activity decreased or was maintained in comparison with the activity of its parent compound (CoA). Nevertheless, both compounds showed higher activity than the extract and, in some case, even higher than the standards. This is in agreement with the results obtained by Heleno et al. ${ }^{5}$ that described a decrease or maintenance of antibacterial activity of protected glucuronide derivatives of $p$-hydroxybenzoic and cinnamic acids. Regarding antifungal activity, CoAGP gave higher effects than its parental phenolic acid; therefore, the inclusion of acetyl groups in the molecule increased the activity of CoA, which is also in agreement with the reported activity of protected glucuronide derivatives of $p$ hydroxybenzoic and cinnamic acids. ${ }^{5}$

Methylated derivatives of $p$-coumaric acid (CoAM1, CoAM2 and CoAM3) revealed higher antimicrobial activity than the parental compound, than the extract and even than the standards used. In most of the cases the antimicrobial activity significantly increased when compared to the activity of the parental compound CoA due to the inclusion of methyl groups in its structure. The opposite was observed for HA and CA that presented higher antimicrobial activity than the correspondent methylated derivatives (HAM1, HAM2 and HAM3, and CAM, respectively). The inclusion of methyl groups in the parental acids did not increase the antimicrobial activity, despite the good results obtained comparing with the extract and with standards.

It should be also highlighted that all the compounds showed much better antifungal than antibacterial activity

The colored conidiophores of some Aspergillus and Penicillium species contains pigments belonging to the group of melanins: a green colored chromoprotein and a black 
insoluble pigment. ${ }^{21}$ Melanin production by fungi contributes to the virulence of pathogens of humans as well as those of food crops. ${ }^{22}$ It was shown that this pigment has an important role in the protection of the fungus against immune effector cells; it is able to scavenge reactive oxygen species generated by alveolar macrophages and neutrophils of the host. ${ }^{23}$ Because melanin is an important factor in fungal virulence, the demelanizing activity of $C$. atramentaria extract and individual compounds was investigated in eight microfungi, the same used for antifungal activity. The results were expressed as minimum demelanizing concentrations (MDC), which were defined as sublethal and subinhibitory concentration necessary to provoke demelanization in fungus during $72 \mathrm{~h}$. Previous studies of demelanization activities of some mushroom extracts (Morchella esculenta and Ganoderma lucidum) showed very strong effect on few microfungi. ${ }^{4,5}$ The organic acid derivatives exhibited very strong antifungal activity, but also demelanizing effect at very low concentrations on three microfungi: Aspergillus niger, A. fumigatus and Penicillium verrucosum var. cyclopium.

\section{Conclusions}

The inclusion of methyl groups in the parental compounds strongly increased the antimicrobial activity of CoA, while the inclusion of acetyl groups increased the antifungal activity but maintained the antibacterial effects. For HA and CA, the inclusion of methyl groups did not increase the antimicrobial activity, but increased the demelanizing activity of the parental acids. As far as we know, this is the first report on the antifungal and demelanizing activity of $C$. atramentaria methanolic extract as well as antibacterial, antifungal and demelanizing activities of $p$-coumaric acid and its 
glucuronide and methylated derivatives, and of $p$-hydroxybenzoic and cinnamic acid methylated derivatives.

\section{Competing interests}

The authors declare no competing financial interest.

\section{Acknowledgements}

The authors are grateful to Fundação para a Ciência e a Tecnologia (FCT, Portugal) for financial support to the Portuguese NMR network and to FCT and FEDERCOMPETE/QREN/EU for the financial support through the research project PTDC/AGR-ALI/110062/2009 and the research centres (PEst-C/QUI/UI0686/2011 and PEst-OE/AGR/UI0690/2011). S.A. Heleno (BD/70304/2010) also thanks FCT, POPHQREN and FSE for her grant. The authors also thank to Serbian Ministry of Education, Science and Technological development (grant number 173032) for financial support.

\section{References}

1. N.K. Ishikawa, Y. Fukushi, K. Yamaji, S. Tahara, K. Takahashi, J. Nat. Prod., 2001, $64,932-934$.

2. M.J. Alves, I.C.F.R. Ferreira, J. Dias, V. Teixeira, A. Martins, M. Pintado, Planta Med., $2012,78,1707-1718$.

3. P. Roupas, J. Keogh, M. Noakes, C. Margetts, P. Taylor, J. Funct. Food., 2012, 4, 687709. 
4. S.A. Heleno, D. Stojković, J. Glamočlija, M. Soković, A. Martins, M.J.R.P. Queiroz, I.C.F.R. Ferreira, Food Res. Int., 2013, 51, 234-243.

5. S.A. Heleno, I.C.F.R. Ferreira, A.P. Esteves, A. Ćirić, J. Glamočlija, A. Martins, M. Soković, M.J.R.P. Queiroz, Food Chem. Toxicol., 2013, 58, 95-100.

6. M.J. Alves, I.C.F.R. Ferreira, J. Dias, V. Teixeira, A. Martins, M. Pintado, Curr. Top. Med., 2013, 13, 2648-2659.

7. L. Barros, R.C. Calhelha, J.A. Vaz, I.C.F.R. Ferreira, P. Baptista, L.M. Estevinho, Eur. Food Res. Technol., 2007, 225, 151-156.

8. B. Dulger, C.C. Ergul, F. Gucin, Fitoterapia, 2002, 73, 695-697.

9. S.A. Heleno, L. Barros, M.J.R.P. Queiroz, C. Santos-Buelga, I.C.F.R. Ferreira, J. Agric. Food Chem., 2012, 60, 4634-4640.

10. S.A. Heleno, I.C.F.R. Ferreira, R.C. Calhelha, A.P. Esteves, A. Martins, M.J.R.P. Queiroz, Food Res. Int., 2014, 55, 170-175.

11. A.R. Rechner, G. Kuhnle, P. Bremner, G.P. Hubbard, K.P. Moore, C.A. Rice-Evans, Free Rad. Biol. Med., 2002, 33, 220-235.

12. D.D. Orhan, B. Özçelik, S. Özgen, F. Ergun, Microbiol. Res., 2010, 165, 496-500.

13. Z. Lou, H. Wang, S. Rao, J. Sun, C. Ma, J. Li, Food Control, 2012, 25, 550-554.

14. M.J. Alves, I.C.F.R. Ferreira, H.C. Froufe, R.M.V. Abreu, A. Martins, M. Pintado, J. Appl. Microbiol., 2013, 115, 346-357.

15. J.A. Oria de Rueda, Hongos y setas. Tesoro de nuestros montes, 2007. Palencia, Spain: Ediciones Cálamo. 
16. Clinical and Laboratory Standards Institute, Methods for dilution antimicrobial susceptibility tests for bacteria that grow aerobically. Approved standard, $8^{\text {th }}$ ed. CLSI publication M07-A8, 2009. Wayne, PA.

17. T. Tsukatani, H. Suenaga, M. Shiga, K. Noguchi, M. Ishiyama, T. Ezoe, K. Matsumoto, J. Microbiol. Method., 2012, 90, 160-166.

18. C. Booth, Fungal culture media. In JR Norris \& DW Ribbons (Eds.), Methods in microbiology, 1971, pp. 49-94. London and New York: Academic Press.

19. A. Espinel-Ingrof, J. Clin. Microbiol., 2001, 39, 1360-1367.

20. C.N. Osuji, E.U. Nwabueze, T.O., Akunna, E.O. Ahaotu, Int. J. Appl. Sci. Eng., 2013, $2,61-65$.

21. H.C. Eismann, A. Casadevall, Appl. Microbiol. Biotechnol., 2012, 93, 931-940.

22. L.H. Rosa, L.M.A. Vieira, I.F. Santiago, C.A. Rosa, FEMS Microbiol. Ecol., 2010, 73, 178-189.

23. A.A. Brakhage, B. Liebmann, Med. Mycol., 2005, 43, S75-S82. 